\title{
Evaluation of sigma value and quality goal index for brain natriuretic peptide test
}

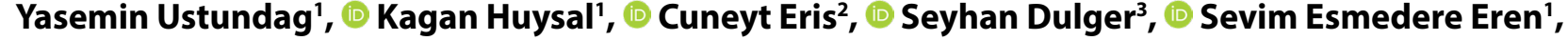 \\ Senol Yavuz ${ }^{2}$
}

'Department of Clinical Biochemistry, Bursa Yuksek Ihtisas Training and Research Hospital, Bursa, Turkey

${ }^{2}$ Department of Cardiovascular Surgery, Bursa Yuksek Ihtisas Training and Research Hospital, Bursa, Turkey

${ }^{3}$ Department of Pulmonary Diseases, Bursa Yuksek Ihtisas Training and Research Hospital, Bursa, Turkey

\begin{abstract}
Objectives: In this study, we aimed to evaluate the analytical process by determining the sigma values and quality goal index (QGI) of the brain natriuretic peptide (BNP) test parameter performed in our emergency laboratory.

Methods: BNP levels were tested using a commercially available immunoassay autoanalyser (Advia Centaur XP; Siemens Healthcare Diagnostic Ltd., Muenchen, Germany). Bias was calculated by comparison with the group mean for each external quality assessment report, and an internal quality control-based approach was applied. The internal quality control results of the BNP test levels between September and December 2018 and external quality program reports were used in the coefficient of variation (CV) and bias calculations. The sigma metrics and QGI were calculated.

Results: The sigma metric calculated from the external quality control was 2.1 and the QGI was 0.34 . In the internal quality control study, the sigma level was 1.92 , and the QGI was 0.55 , as calculated at the $400 \mathrm{pg} / \mathrm{ml}$ level. The QGI level suggests that the problem in the BNP study was imprecision.

Conclusion: Unsatisfactory sigma levels for BNP tests were achieved using both methods of calculating sigma metrics. Keywords: Brain, natriuretic peptide, quality control, six sigma
\end{abstract}

$\mathrm{T}^{\mathrm{s}}$ he b-type or brain natriuretic peptide (BNP) is a member of the natriuretic peptide family that is involved in many biological events in the body, including regulation of blood pressure and blood volume [1-3]. BNP is mainly synthesized in the cardiac ventricle as proBNP, which is a prohormone consisting of 108 amino acids. ProBNP is cleaved in the circulation as an active hormone 32 amino acid structure that corresponds to the C-terminus of proBNP. The $\mathrm{N}$ terminal (NT) 76 amino acid NT-proBNP is an inactive protein. Both BNP and NT-proBNP have a wide range of clinical effects that increase cardiac stress and output [1-3].

Measurement of BNP levels to differentiate the causes of acute dyspnoea in patients admitted to the emergency de- partment has high diagnostic accuracy $[3,4]$. Heart failure (HF) is unlikely when the plasma BNP level is $<100 \mathrm{pg} / \mathrm{ml}$, and a level higher than $400 \mathrm{pg} / \mathrm{ml}$ is appropriate for ruling in acute HF, which makes it a suitable test for patient identification in emergency care settings [1-4]. However, recent studies suggested that there are significant differences in analytical performance among commercial methods for BNP immunoassays [5]

An erroneous result, in which the difference between a test result and the corresponding true value exceeds the allowable total error (Tea), leads to medically significant errors. To improve patient safety, zero errors should be targeted in health services that cannot be possible in a real laboratory setting [6].

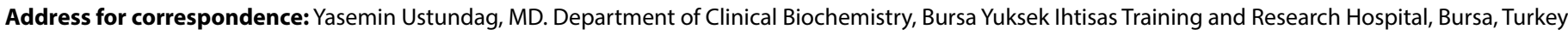
Phone: +90 5324823692 E-mail: yaseminbudak2000@yahoo.com ORCID: 0000-0003-2415-0372

Submitted Date: January 11, 2020 Accepted Date: June 17, 2020 Available Online Date: October 06, 2020

${ }^{\circ}$ Copyright 2020 by International Journal of Medical Biochemistry - Available online at www.internationalbiochemistry.com

OPEN ACCESS This work is licensed under a Creative Commons Attribution-NonCommercial 4.0 International License. 
The total number of errors made by the laboratory can be measured using the Six Sigma metric method [6-9]. The process sigma level signifies the error rate; higher sigma values show less error, and a sigma level of 6 or greater indicates world-class performance (3.4 errors per million) [6]. The minimum sigma level calculated must be at least 3 in the analytical process for laboratory performance evaluation $[6,10]$. In the case of process sigma levels $<3$ (error rate higher than $2700 / 106)$, improving the performance of the testing methods should be tried [11].

Different approaches to bias and imprecision calculation may influence the final sigma calculation. In most studies, external quality control (EQC) reports were used in bias calculation and the coefficient of variation (CV) was obtained from internal quality control (IQC) studies for the sigma calculation [7-9]. EQC results provide information on analytical performance across laboratories [12]. However, the concentrations of IQC materials may differ significantly from the EQC samples used in bias calculation.

Considering that the IQC samples typically have three different concentration levels and that it is easier to cover different levels of analysis for medical decision making, the use of IQC in the calculation of bias in the sigma calculation evaluates different concentrations of sigma properly [13]. On the other hand, it is suggested that the use of the IQC target value in bias calculation is not appropriate because it is not clear how close they are to the real value [14].

The quality goal index (QGI) can be used if the sigma level is low, to determine the extent to which both bias and imprecision meet the quality objectives [15].

In this study, we aimed to evaluate the analytical process by determining the sigma values and QGI of the BNP test parameter performed in our emergency laboratory. Bias was calculated by comparison with the group mean for each external quality assessment report, and an internal quality control-based approach was applied.

\section{Materials and Methods}

This retrospective study was conducted in Bursa Yuksek Ihtisas Training and Research Hospital emergency laboratory that provides a clinical laboratory service to the 1430-bed hospital. Ethical approval was obtained from the Ethics Committee of our institution (2011-KAEK-25 2019/01-04).
BNP levels were tested using a commercially available immunoassay autoanalyser (Advia Centaur XP; Siemens Healthcare Diagnostic Ltd., Germany). Reagents, calibrators and internal quality control sera were supplied by the manufacturer. The calibrations were carried out in accordance with the relevant package insert instructions and were in two points (Lot 60417A67 Siemens Healthcare Diagnostic Inc. USA) (0 pg/ml and $1273 \mathrm{pg} / \mathrm{ml}$ ).

The internal quality control results of the BNP test between September and December 2018 were obtained from the laboratory computer system. The internal quality control materials were three levels (Lots 3816491, 3816492 and 3816493; Siemens Healthcare Diagnostic Inc., USA) (Table 1). The IQC is run daily, and the CV percentage for this study was calculated from the results of internal quality control.

In the bias calculation, we used the EQC reports produced by the External Quality Programme (KBUDEK) of the Clinical Biochemistry Specialists Association, Istanbul, Turkey. The EQC results for the months of September to December 2018 (four EQC results) were used, and the average was calculated.

The formula used when making calculations was as follows:

Bias (\%) EQC=(our mean-mean of all laboratories using the same instrument and method)/(mean of all laboratories using the same instrument and method $) \times 100$

The bias was also obtained from reagent package inserts based on the target value, averaged from the Siemens global report for the same assay performed with the same instrument and method.

Bias (\%) IQC=(our mean-target value from the package insert)/(target value from the package insert) $\times 100$

IQC results were used in the percentage CV calculation. The averages and standard deviations were calculated using SPSS version 21 (SPSS Inc., Chicago, IL, USA).

The CV was calculated using the following formula: CV $(\%)=($ standard deviation $\times 100) /$ laboratory mean $($ IQC)

The TEa value for BNP values above $100 \mathrm{pg} / \mathrm{ml}$ was $20 \%$, taken from the American Association of Bioanalysts (AAB) and Royal College of Pathologists of Australasia (RCPA) [16].

The Sigma levels were calculated using the following formulas:

Process Sigma1 $=($ Tea\%-biasEQC\%)/CVIQC\%

Process Sigma2=(Tea\%-biasIQC\%)/CVIQC\%

Table 1. The \% coefficient of variation of BNP internal quality control for September to December 2018

\begin{tabular}{|c|c|c|c|c|c|c|c|}
\hline & $\begin{array}{l}\text { Assigned } \\
\text { target value }\end{array}$ & $\begin{array}{l}\text { Measured min-max } \\
\text { value }(\mathrm{pg} / \mathrm{ml})\end{array}$ & Mean \pm SD & BiasIQC (\%) & CV (\%) & SigmalQC & QGI \\
\hline QC Level 1 (3816491) & 51.9 & $39.0-55.0$ & $44.7 \pm 3.6$ & 13.87 & 8.05 & 0.76 & 1.14 \\
\hline QC Level 2 (3816493) & 1848 & $1538-2060$ & $1753 \pm 126$ & 5.14 & 7.18 & 2.06 & 0.48 \\
\hline
\end{tabular}

QC: Quality control, CV: Coefficient of variation, Min: minimum, Max: maximum, IQC: Internal quality control, Process SigmalQC=(\% Tea- $\%$ biasIQC) $/ \%$ CVIQC 


\begin{tabular}{lllll} 
Table 2. External quality assessment data & & \\
\hline Period & $\begin{array}{l}\text { Lab result } \\
\text { (pg/ml) }\end{array}$ & $\begin{array}{l}\text { Mean of the } \\
\text { group }(\mathbf{p g} / \mathbf{m l})\end{array}$ & Bias (\%) & SDI \\
& 189.53 & 183.57 & 3.2 & 0.84 \\
\hline September & 2571.50 & 2627.08 & 2.1 & -0.23 \\
October & 160.63 & 175.48 & 8.5 & -0.67 \\
November & 2789.00 & 2837.68 & 1.7 & -0.26 \\
December & 27.06 &
\end{tabular}

Lab: Laboratory, SDI: Standard deviation index

The QGI was calculated using the following formula:

$\mathrm{QGl}=$ Bias $/ 1.5 \mathrm{XCV}$

A QGI value of $<0.8$ indicates that the precision of the measurement procedure needs improvement; QGI values of $>1.2$ indicate that the trueness needed to be improved, and values of $0.8 \leq \mathrm{QGl} \leq 1.2$ indicated that both the precision and trueness needed to be improved [17].

\section{Results}

We used a three-level internal quality control sample with means of $44.7 \pm 3.6 \mathrm{pg} / \mathrm{ml}, 468 \pm 34 \mathrm{pg} / \mathrm{ml}$, and $1753 \pm 126 \mathrm{pg} /$ $\mathrm{ml}$, and the calculated CVs were $8.05 \%, 7.26 \%$ and $7.18 \%$, respectively (Table 1 ). The average CV was calculated as $7.49 \%$.

The average biasEQC of BNP test results was calculated as $3.87 \%$ (Table 2). The SDI was acceptable in each EQC result as $<0.125$ (Table 2). The target SDI score was 0.0 , which indicated that there was no difference between the laboratory mean and target mean [18].

The sigma metric calculated from the EQC was 2.1, and the QGI was 0.34.

We also calculated sigma metrics from the internal quality control study. The sigma level was 0.76 to 2.06 in different quality control levels. The QGI levels suggested that the problem in the BNP study was imprecision (Table 1).

\section{Discussion}

When evaluated on the sigma scale, we obtained a low sigma when the bias was taken from the external quality control reports of the BNP test under our emergency laboratory conditions. Since $400 \mathrm{pg} / \mathrm{ml}$ is the medical decision level, higher-quality performance at this level should be aimed for. We found a sigma value of $<3$, according to the bias we obtained with the difference from the internal quality control target value at all levels [13].

Bias and precision both influence the Sigma metrics while precision has a considerable impact [19]. A few studies have evaluated both Sigma metrics and QGI levels for the analytic process $[20,21]$.

QGI levels for the BNP test in our emergency laboratory conditions lower than 0.8 are due to high imprecision rather than a bias problem that is difficult to correct.
The percentage $\mathrm{CV}$ for monitoring the imprecision of measurements reflects the quality of the laboratory analysis phase [22]. In a study of 72 laboratories in China, the findings showed that using the Siemens BNP kits, the median CV ranged from $4.87 \%$ (interquartile range [IQR] 2.38) to $5.17 \%$ (IQR 3.33) [23]. In the manufacturers' datasheet, the precision CV between days was reported to vary between 2.9 and $3.3 \%$. In our study, the precision CV was between $7.18 \%$ to $8.05 \%$. The CV percentages we obtained with three different IQC materials in our laboratory conditions were not comparable with the claims of the kit producer. The $\mathrm{CV}$ in the kit datasheet was realised in a shorter time and in an ideal environment; in this study, we took a four-month IQC study for precision calculation.

There are several quality requirements for the evaluation of imprecision, such as $1 / 3$ total allowable error (TEa), $1 / 4 \mathrm{TEa}$, and the specifications based on biological variation, including the minimal $(0.25 \mathrm{CVl}$. CVl, the intrasubject biological variation), desirable $(0.50 \mathrm{CVI})$ and optimal $(0.75 \mathrm{CVI})$ allowable imprecision [22, 24].

Biological variation of plasma BNP concentrations is quite large in both healthy patients and patients with heart failure because BNP half-life has short secretory bursts [25-27]. For the BNP precision with an individual biological variation of $30 \%$, the desirable analytical CV target is $15 \%$ (i.e., should be equal to half of the inter-individual variation) [24]. However, the consensus for the clinical use of BNP tests advocates that total imprecision should be as low as possible and $<10 \%$ [28]. In particular, it is desirable to minimise the analytical sensitivity component to monitor treatment with serial BNP measurements in clinical situations [28]. The best imprecision is able to detect significantly narrower changes in BNP concentrations.

TEa is used to define the acceptable analytical performance of the device, validate quality control and compare results for analytes measured in different systems [29]. In recent studies, a consensus has been reached on the use of $1 / 3$ 1/TEa and $1 / 4$ TEa as a target to evaluate the measurement systems' imprecision among clinical laboratory experts [30-32]. The findings obtained in this study showed that the BNP test did not meet $5 \%$ of the imprecision with $1 / 4 \mathrm{TEa}$. The application of more stringent internal quality control rules by clinical laboratories can improve the precision performance of the BNP assay by reducing CV levels [29]. Meanwhile, to achieve a sigma performance level of at least 6 while assuming zero bias, the maximum allowable CV was 3.3\% using 20\% TEa taken from the $A A B$ and the RCPA.

Determining the optimal control frequency is currently different between laboratories. In our laboratory, we perform three levels of quality control once a day. Burnett et al. offer a maximum QC of three levels, three times a day for tests under sigma level 3, and consider testing specimens in duplicate [30]. However, this will increase the cost per unit.

In our study, sigma metrics calculated with bias from internal quality control did not perform better than predicted with ex- 
ternal quality control. This can be explained by different factors, such as storage, freezing and stability problems, which can be considered specific to the manipulation of the internal quality control material.

\section{Limitations}

The BNP test was not compared to a reference method. The external quality control programme in which we participated was based on a peer group rather than an accuracy programme.

\section{Conclusion}

Our results showed that unsatisfactory sigma levels were achieved using both the IQC and EQC methods of calculating sigma. The imprecision of the measurement is not desirable. Thus, we must choose appropriate actions, such as strict monitoring, as well as increased frequency of IQC runs to change this situation. Laboratories should strive to reduce laboratory errors to a negligible level.

\section{Conflict of interest: None declared.}

Ethics Committee Approval: Ethical approval was obtained from the Ethics Committee of our institution (2011-KAEK-25 2019/01-04).

Financial Disclosure: None declared.

Peer-review: Externally peer-reviewed.

\section{References}

1. Fu S, Ping P, Wang F, Luo L. Synthesis, secretion, function, metabolism and application of natriuretic peptides in heart failure. J Biol Eng 2018;12:2. [CrossRef]

2. Zhang $Z L$, Li R, Yang FY, Xi L. Natriuretic peptide family as diagnostic/prognostic biomarker and treatment modality in management of adult and geriatric patients with heart failure: remaining issues and challenges. J Geriatr Cardiol 2018;15(8):540-6.

3. Korenstein D, Wisnivesky JP, Wyer P, Adler R, Ponieman D, McGinn T. The utility of B-type natriuretic peptide in the diagnosis of heart failure in the emergency department: a systematic review. BMC Emerg Med 2007;7:6.

4. Krupicka J, Jonata T, Hradec J. Natriuretic peptides in heart failure. Cor et Vasa 2013;55:370-6. [CrossRef]

5. Clerico A, Franzini M, Masotti S, Prontera C, Passino C. State of the art of immunoassay methods for B-type natriuretic peptides: An update. Crit Rev Clin Lab Sci 2015;52(2):56-69.

6. Westgard S, Bayat H, Westgard JO. Analytical Sigma metrics: A review of Six Sigma implementation tools for medical laboratories. Biochem Med (Zagreb) 2018;28(2):020502. [CrossRef]

7. Ustundag BY, Huysal K. Application of Sigma Metrics and Performance Comparison Between Two Biochemistry Analyser and a Blood Gas Analyser for the Determination of Electrolytes. J Clin Diagn Res 2017;11(2):BC06-BC09.
8. Huysal K, Budak YU. Application of sigma metrics for the assessment of quality assurance using the MQ-2000 PT HbA1C analyzer. Biochem Med (Zagreb) 2015;25(3):416-20. [CrossRef]

9. Kumar BV, Mohan T. Sigma metrics as a tool for evaluating the performance of internal quality control in a clinical chemistry laboratory. J Lab Physicians 2018;10(2):194-9. [CrossRef]

10. Westgard JO, Westgard SA. The quality of laboratory testing today: an assessment of sigma metrics for analytic quality using performance data from proficiency testing surveys and the CLIA criteria for acceptable performance. Am J Clin Pathol 2006;125(3):343-54. [CrossRef]

11. Schoenmakers $\mathrm{CH}$, Naus AJ, Vermeer HJ, van Loon D, Steen $G$. Practical application of Sigma Metrics QC procedures in clinical chemistry. Clin Chem Lab Med 2011;49(11):1837-43. [CrossRef]

12. Jones GR, Sikaris K, Gill J. Allowable Limits of Performance for External Quality Assurance Programs - an Approach to Application of the Stockholm Criteria by the RCPA Quality Assurance Programs. Clin Biochem Rev 2012;33(4):133-9.

13. Guo X, Zhang T, Gao X, Li P, You T, Wu Q, et al. Sigma metrics for assessing the analytical quality of clinical chemistry assays: a comparison of two approaches: Electronic supplementary material available online for this article. Biochem Med (Zagreb) 2018;28(2):020708. [CrossRef]

14. Hens K, Berth M, Armbruster D, Westgard S. Sigma metrics used to assess analytical quality of clinical chemistry assays: importance of the allowable total error (TEa) target. Clin Chem Lab Med 2014;52(7):973-80. [CrossRef]

15. Schweikhart SA, Dembe AE. The applicability of Lean and Six Sigma techniques to clinical and translational research. J Investig Med 2009;57(7):748-55. [CrossRef]

16. Royal College of Pathologists of Australasia (RCPA). Allowable Limits of Performance for Biochemistry. Available at: http:// www.rcpaqap.com.au/docs/2014/chempath/ALP.pdf. Accessed Mar 20, 2019.

17. Parry DM. Quality goal index. Available at: https://www.westgard.com/guest34.htm Accessed Mar 20, 2019.

18. Yerram S, Sripad DV, Prabodh VS. External Quality Assurance Scheme (EQAS): Criteria for evaluating performance of a laboratory. IOSR J Biotechnol Biochem 2018:4(4);16-20.

19. Hyltoft Petersen P, Klee GG. Influence of analytical bias and imprecision on the number of false positive results using Guideline-Driven Medical Decision Limits. Clin Chim Acta 2014;430:1-8.

20. Verma M, Dahiya K, Ghalaut VS, Dhupper V. Assessment of quality control system by sigma metrics and quality goal index ratio: A roadmap towards preparation for NABL. World J Methodol 2018;8(3):44-50. [CrossRef]

21. Varela B, Pacheco G. Comprehensive evaluation of the internal and external quality control to redefine analytical quality goals. Biochem Med (Zagreb) 2018;28(2):020710. [CrossRef]

22. Fei Y, Wang W, He F, Zhong K, Wang Z. Imprecision Investigation and Analysis of Routine Chemistry in China. J Clin Lab Anal 2016;30(5):444-50. [CrossRef]

23. Sun $\mathrm{H}$, Wang W, Zhao H, Zhang C, He F, Zhong K, et al. Internal quality control status for BNP and NT-proBNP in China from 2014 to 2017. J Clin Lab Anal 2019;33(1):e22643. [CrossRef] 
24. Petersen PH, Fraser CG, Jørgensen L, Brandslund I, Stahl M, Gowans $\mathrm{E}$, et al. Combination of analytical quality specifications based on biological within- and between-subject variation. Ann Clin Biochem 2002;39(Pt 6):543-50. [CrossRef]

25. Wu AH, Smith A. Biological variation of the natriuretic peptides and their role in monitoring patients with heart failure. Eur J Heart Fail 2004;6(3):355-8. [CrossRef]

26. Pagani F, Stefini F, Pangethini M. Biological variation in serum concentrations of $\mathrm{N}$-terminal pro-brain natriuretic peptide. Clin Chem 2003;49(Suppl):A34.

27. Bruins S, Fokkema MR, Römer JW, Dejongste MJ, van der Dijs $\mathrm{FP}$, van den Ouweland JM, et al. High intraindividual variation of B-type natriuretic peptide (BNP) and amino-termi- nal proBNP in patients with stable chronic heart failure. Clin Chem 2004;50(11):2052-8. [CrossRef]

28. Apple FS, Panteghini M, Ravkilde J, Mair J, Wu AH, Tate J, Pagani F, Christenson RH, Jaffe AS; Committee on Standardization of Markers of Cardiac Damage of the IFCC. Quality specifications for B-type natriuretic peptide assays. Clin Chem 2005;51(3):486-93.

29. Mao X, Shao J, Zhang B, Wang Y. Evaluating analytical quality in clinical biochemistry laboratory using Six Sigma. Biochem Med (Zagreb) 2018;28(2):020904. [CrossRef]

30. Burnett D, Ceriotti F, Cooper G, Parvin C, Plebani M, Westgard J. Collective opinion paper on findings of the 2009 convocation of experts on quality control. Clin Chem Lab Med 2010;48(1):41-52. [CrossRef] 